\title{
Adaptive Control of Shell and Tube Heat Exchanger
}

\author{
I.Thirunavukkarasu ${ }^{1}$ Mithun.P ${ }^{2}$ V.I.George ${ }^{3 *}$ Shreesha. $C^{4}$
}

\begin{abstract}
This paper deals with the temperature control of a shell and tube heat exchanger(STHE) using adaptive control. The parameters of the process are identified using Recursive least square algorithm. The control algorithm is based on the Linear Quadratic control method and the parameters of the controller are computed based on the pole placement method. 2DoF control structure is used for the controlling of process.
\end{abstract}

Keywords-Adaptive control, Shell and tube heat exchanger, Servo response, Real time experimentation.

\section{Introduction}

Heat exchangers are devices that facilitate the exchange of heat between two fluids that are different temperature while keeping them from mixing with each other. Heat exchangers are mainly used in chemical processing and power production plants. Since they are relatively easy to use experimentally and they show nonlinear and non-stationary properties, the heat exchangers are frequently used as a demonstration of control. In this paper an attempt is made to design an adaptive controller to control the outlet temperature of pilot scale heat exchanger. The experimental heat exchanger setup is shown in Fig.1.

In adaptive control, the control is divided into two parts. First part is the identification of the process parameter. The Recursive least square algorithm with directional forgetting factor is used for the identification. The second part is the computing of controller parameter and output of the controller. The linear quadratic method is used as the control strategy. In this the controller parameter is computed based on the process parameter and update the controller parameter. Then based on the updated parameters of the controller control signal is computed. This control signal is given to the process.

Section-2 discusses about the STHE, Section-3 deals about the control methodology, Section-4 deals with the parameter estimation of dynamic controller. Section-5 shows the adaptive controller tuning and results are discussed in Section6.

1-Associate Professor 2- PG Student M.Tech(CS)

3 \& 4 - Professor, Instrumentation and Control Engineering, Manipal Institute of Technology, Manipal University, Manipal-576 104, Karnataka, India

Corresponding Author Mail:

\section{Shell and Tube Heat Exchanger}

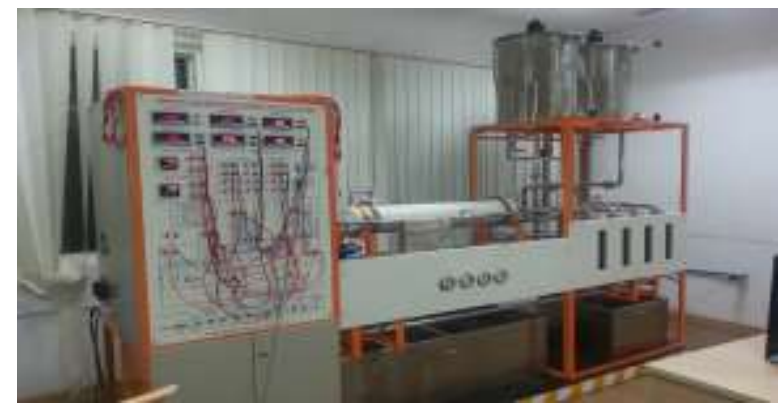

Fig(1) Setup of the physical experimentation system.

Shell and tube heat exchangers (STHE) are probably the most common type of heat exchangers applicable for a wide range of operating temperatures and pressures .The shell and tube heat exchanger has hot water in the tube side and cold water in the shell side. Heat energy is transfered fromhot water to the cold water. The outlet temperature of the hot water is controlled by varying the cold water flow rate. So that outlet hot water temperature is the controlled variable and inlet cold water flow rate is the manipulated variable.

\section{Control Methodology}

The adaptive control is shown in Fig.(2) The parameters of the process are find out by recursive least square algorithm using input and output of the process.

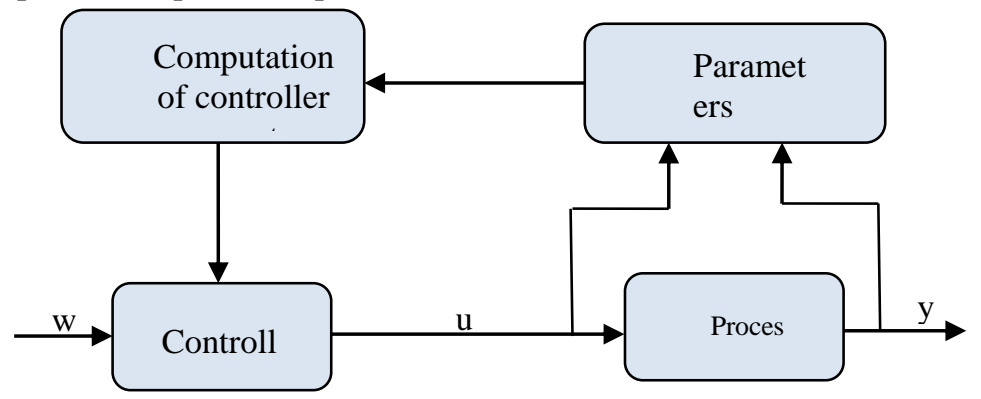

Fig(2) Control methodology

The parameters of the Then by using these parameters the controller parameters are calculated and updated. 


\section{Parameter Identification:}

The plant has the transfer function has the form [2,3]

$$
\frac{b_{1} s+b_{2}}{s^{2}+a_{1} s+a_{2}}
$$

Its discrete form is given by eqn (2)

$$
\frac{b_{1} z^{-1}+b_{2} z^{-2}}{1+a_{1} z^{-1}+a_{2} z^{-2}}
$$

The parameter of the plant $\mathrm{b} 1, \mathrm{~b} 2, \mathrm{a} 1, \mathrm{a} 2$ is estimated using

Recursive least square algorithm with directional forgetting.

The ARX model of the plant is given by

$$
y(k)=\theta^{T}(k) \phi(k-1)+e_{s}(k)
$$

Where

$$
\theta^{T}(k)=\left[a_{1}, a_{2}, b_{1}, b_{2}\right]
$$

is the vector of parameters of the model

$$
\phi(k-1)=[-y(k-1),-y(k-2), u(k-1), u(k-2)]
$$

is the

data vector, so-called regressor.The task is to estimate recursively the unknown parameters $\theta$ of model on the basis of inputs and outputs at time $\mathrm{k}$

$\{u(i), y(i), i=k, k-1, k-2, \ldots, 0\}$. Therefore, the unknown vector parameter $\theta$ can be found when minimizing the criterion.

$$
J_{k}(\theta)=\sum_{i=0}^{k} e_{s}^{2}(i)
$$

Exponential forgetting can be used for monitoring the slow changes in the parameter of the identified process. Then, it is necessary to minimize the modified criterion

$$
J_{k}(\theta)=\sum_{i=0}^{k} \varphi^{2(k-i)} e_{s}^{2}(i)
$$

where $0<\varphi^{2} \leq 1$ is the exponential forgetting factor. The vector of parameter estimates is updated according to the recursiverelation

$$
\hat{\theta}(k)=\hat{\theta}(k-1)+\frac{C(k) \phi(k-1)}{1+\xi(k)} \hat{e}(k)
$$

Where

$$
\xi(k)=\phi^{T}(k-1) C(k) \phi(k-1)
$$

is an auxiliary scalar and

$$
\hat{e}(k)=y(k)-\hat{\theta}^{T}(k-1) \phi(k-1)
$$

is the prediction error.If $\xi(k)>0$, the square covariance matrixis updated by relation,

$$
C(k)=C(k-1)-\frac{C(k-1) \phi(k-1) \phi^{T}(k-1) C(k-1)}{\varepsilon^{-1}(k)+\xi(k)}
$$

Where

$$
\varepsilon(k)=\varphi(k)-\frac{1-\varphi(k)}{\xi(k-1)}
$$

\section{Adaptive Controller:}

The 2DoF control structure is shown in Fig.3.The controller has the transfer function

$$
Q=\frac{q_{0}+q_{1} z^{-1}}{p_{0}+p_{1} z^{-1}}, R=\frac{r_{0}+r_{1} z^{-1}}{p_{0}+p_{1} z^{-1}}
$$

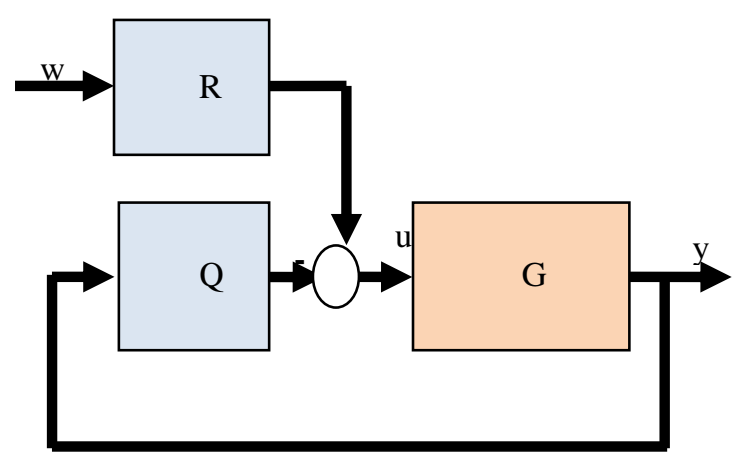

Fig 3. Control system structure

$\mathrm{G}$ is the plant with transfer function given in Equ. (1).

Here the control algorithm is developed based on the linear quadratic method. Linear quadratic control (LQ) methods [4] try to minimize the quadratic criterion by penalizing the controller output

$$
J=\sum_{k=0}^{\infty}\left\{[w(k)-y(k)]^{2}+q_{u}[u(k)]^{2}\right\}
$$

Where $q_{u}$ is the so called penalization constant, $w(k)$ is the reference signal, $y(k)$ is the output and the $u(k)$ is input signal. 
The characteristic equation of the system has the form

$$
A\left(z^{-1}\right) P\left(z^{-1}\right)+B\left(z^{-1}\right) Q\left(z^{-1}\right)=D\left(z^{-1}\right)
$$

The polynomial $D\left(z^{-1}\right)$ is the result of spectral factorization according to the equation,

$$
A\left(z^{-1}\right) q_{u} A(z)+B\left(z^{-1}\right) B(z)=D\left(z^{-1}\right) \delta D(z)
$$

$\delta$ is a constant.

While performing the spectral factorization of a polynomial of the second degree $M\left(z^{-1}\right)$, the following equation is solved

$$
M\left(z^{-1}\right) M(z)=D\left(z^{-1}\right) \delta D(z)
$$

The product of the polynomial can be extended as

$$
\begin{aligned}
& m_{0}+m_{1}\left(z+z^{-1}\right)+m_{2}\left(z^{2}+z^{-2}\right)= \\
& \delta\left(1+d_{1}^{2}+d_{2}^{2}\right)+\delta d_{1}\left(1+d_{2}\right)\left(z+z^{-1}\right)+\delta d_{2}\left(z^{2}+z^{-2}\right)
\end{aligned}
$$

Where the constants of the factorized polynomial on the left side of the equation are combined into coefficients $m_{0}, m_{1}, m_{2}$.

From equations (14)\&(17)

$$
\begin{aligned}
& m_{0}=q_{u}\left(1+a_{1}^{2}+a_{2}^{2}\right)+b_{1}^{2}+b_{2}^{2} \\
& m_{1}=q_{u}\left(a_{1}+a_{1} a_{2}\right)+b_{1} b_{2}
\end{aligned}
$$

$$
m_{2}=q_{u} a_{2}
$$

The parameters of the polynomial D are computed according to equations

$$
\lambda=\frac{m_{0}}{2}-m_{2}+\sqrt{\left(\frac{m_{0}}{2}+m_{2}\right)}-m_{1}^{2}
$$

$$
\delta=\frac{\lambda+\sqrt{\lambda^{2}-4 m_{2}^{2}}}{2}
$$

$$
d_{1}=\frac{m_{1}}{\delta+m_{2}}
$$

$$
d_{2}=\frac{m_{2}}{\delta}
$$

and $d_{0}$ is chosen as 1 .

Then the controller parameter can be obtained from the solution of matrix

$$
r_{0}=\frac{\left(d_{0}+d_{1}+d_{2}+d_{3}\right)}{\left(b_{1}+b_{2}\right)}
$$

$$
\begin{gathered}
r_{1}=0 \\
{\left[\begin{array}{cccc}
1 & 0 & 0 & 0 \\
a_{1} & 1 & b_{1} & 0 \\
a_{2} & a_{1} & b_{2} & b_{1} \\
0 & a_{2} & 0 & b_{2}
\end{array}\right]\left[\begin{array}{l}
p_{0} \\
p_{1} \\
q_{0} \\
q_{1}
\end{array}\right]=\left[\begin{array}{l}
d_{0} \\
d_{1} \\
d_{2} \\
d_{3}
\end{array}\right]}
\end{gathered}
$$

Where $\mathrm{d} 3=0$ and

\section{Results}

The simulation studies was carried out in the MATLABSimulink 2015® environment. The Simulink diagram is shown in Fig.4.

In lab environment the real time experimentation was carried with the shell and tube heat exchanger process. The heat 
exchanger model is assumed to be with the four parameters namely a1,a2,b1 and b2 as given in Equ.1. During the

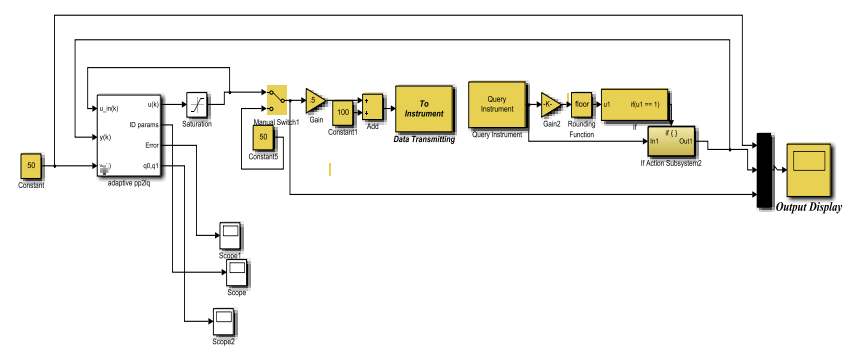

Fig.(4) Simulink diagram for the adaptive control in MATLAB-Simulink 2015®

operation the cold water and hot water temperature will vary slightly and the model will be perturbing with small parameter variations. These parameter variations were measure and plotted in the following figures from Fig.5 to Fig.8 respectively for the parameters a1,a2,b1 and b2.

Fig (5) Change in the values of the parameter a1

Fig (6) Change in the values of the parameter a2

Fig (7) Change in the values of the parameter b1

Fig (8) Change in the values of the parameter b2
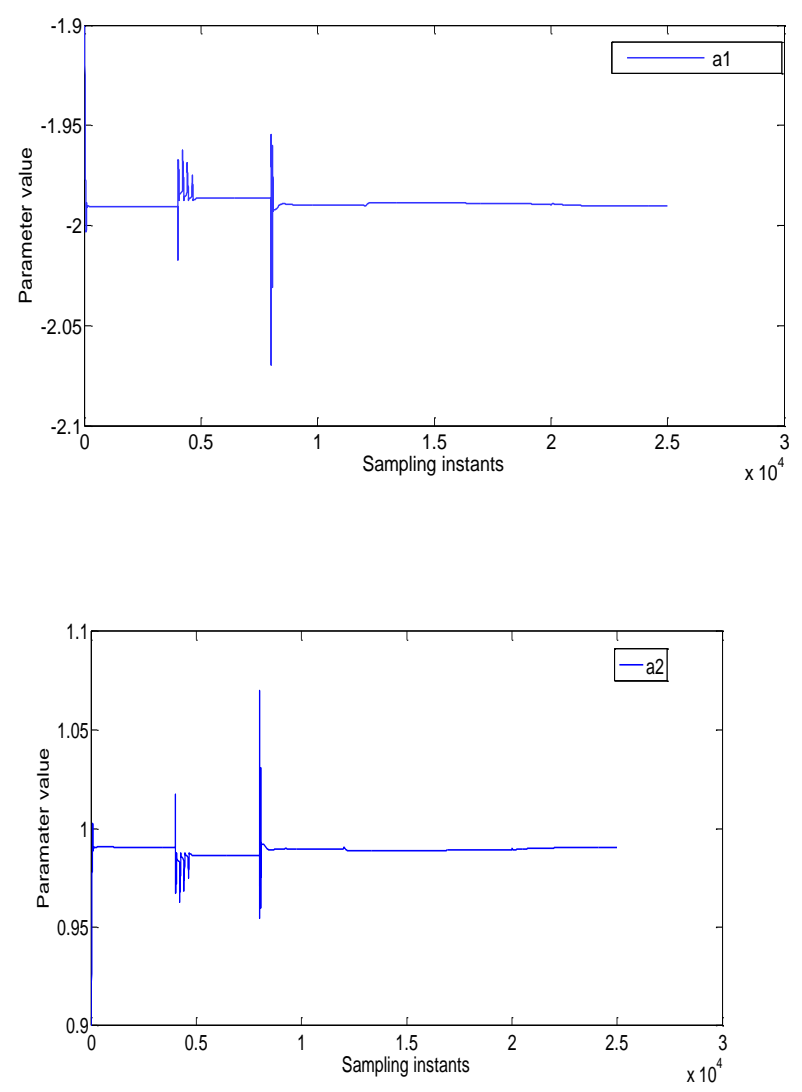

heat exchanger process with the adaptive control with different setpoint operations along with the manipulated variable.

Fig.9. Closed loop response of the heat exchanger process with adaptive control.

Controller output shown in Equ.13 is adaptive in characteristics for the dynamic plant and it is fed to the actuator for corrective action required.

\section{Conclusion}

In this paper an attempt has been made for controlling a dynamically varying process with a dynamic controller. The mathematical model of the heat exchanger given in the equ.

(1) will vary dynamically based on the input hot water and
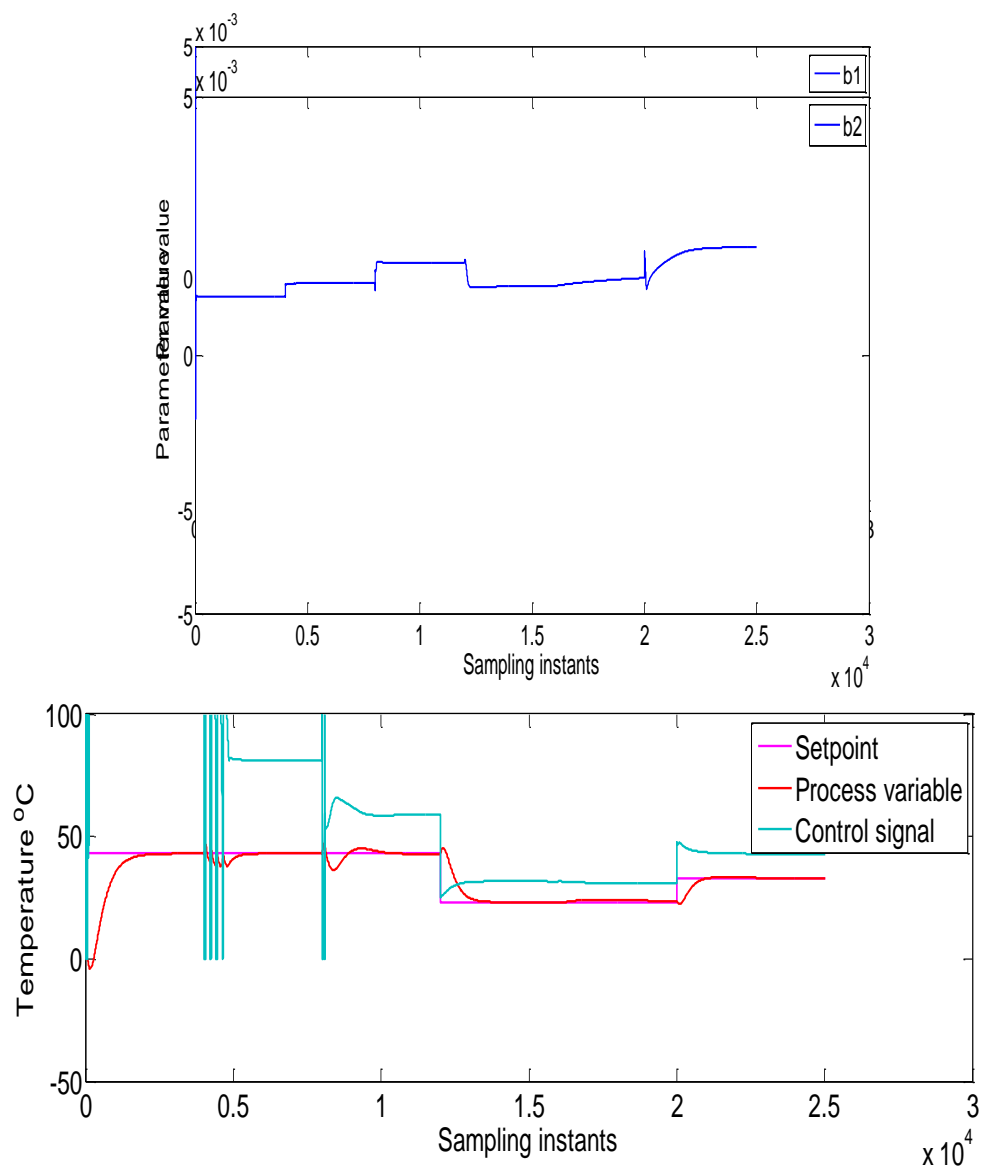

cold water flow rate. So this makes the mathematical model of the heat exchanger vary with time i.e dynamic model.Adaptive controller based on LQ method provides improves the tracking 
response in all time irrespective of the plant parameter variation ( $\mathrm{a} 1, \mathrm{a} 2, \mathrm{~b} 1$ and $\mathrm{b} 2)$. With respect to the change in the plant parameter variation the controller parameters also vary (p0,p1, q0, r0). This makes the controller adaptive with dynamic process and it will give better response from the controller .Fig. 4 to Fig. 8 shows the plant parameter variation happened with respect to time. Figure 9 shows the output response of the heat exchanger for the set point and parameter changes. The summation of the controller parameters action is shown in the Fig.9 which oscillates between 0-100\% initially and reaches steady state action around 50\%. The earlier work of authors in [2] has the real time results of IMC and Robust 2DOF controllers. There the controller works satisfactorily only for the static plant. In this paper the work was extended for the dynamic plant.

\subsection{Observations and Future Work}

An attempt has been made to implement the above given algorithm in the industrial type shell and tube heat exchanger in our PC based Instrumentation lab. Since the process is very slow and the controller doesn't have the integral action the tracking in real time is not satisfactory. Hence planned to implement the same algorithm in the pressure process station compatible of interfacing with MATLAB-2015® is under process and the simulation results are found satisfactory.

\section{ACKNOWLEDGEMENT}

The authors expresses their heart full thanks to Dept. of Instrumentation and Control Engineering, MIT, Manipal University, Karnataka - India for providing us the real time experimental facility to carry out this work and also for providing access to online journal and articles.

\section{References}

[1] Bobál, V., J. Böhm, J. Fessl, and J. Macháček. 2005. Digital self-tuning controllers, Springer Verlag, Berlin, 2005.

[2] Satheeshbabu.R, Dr.I.Thirunavukkarasu et al., "Temperature control of a shell and tube heat exchanger using PID algorithms", International Journal of Advancements in Electronics and Electrical Engineering - IJAEE, Vol.3, Issue.3, Sep.2014.
[3] Dostál, P. J. Vojtěšek, and V. Bobál.” Adaptive LQ control of a shell and tube heat exchanger" International journal $\mathrm{f}$ mathematics and computer in simulation, vol. 7,2013.

[4] Marek Kubalčík and V. Bobál, "Applied continuous- time self - tuning control" Latest trends on systems (Volume II), 2012. ISSN: 1792-4235. 